\title{
Ains/au contraire de la cooccurrence à la concurrence ${ }^{1}$
}

\author{
Monia Mokni \\ Unité de Recherche Syntaxe, Sémantique et Pragmatique \\ Faculté des Lettres et des Sciences Humaines, Sousse, Tunisie \\ mokmonia@yahoo.fr
}

\begin{abstract}
Nous portons notre intérêt, dans ce travail, sur la disparition de l'ancienne conjonction de coordination ains $^{2}$. Nous avons voulu comprendre pourquoi le français se passe d'un coordonnant comme ains, coordonnant qui s'est spécialisé, dès l'ancien français (désormais AF) dans l'expression forte de l'adversativité. Par ailleurs, si nous considérons la disparition de ains comme une perte peut-on dire que cette perte a été compensée ?
\end{abstract}

En nous situant dans le prolongement des travaux de Marchello-Nizia sur la grammaticalisation et le changement linguistique (en particulier 2006), nous proposons de considérer cette disparition comme un maillon dans une chaîne de changements liés qui se sont produits en parallèle avec l'éclipse progressive de ce coordonnant. En effet, selon Marchello-Nizia (2006: 103) «La disparition des unités ou de règles linguistiques n'apparaît généralement que comme un épiphénomène, conséquence d'un autre fait de changement. » Ces changements sont jusque-là considérés comme des phénomènes isolés et sans relation avec cette disparition. Il s'agit surtout de la spécialisation de mais dans son emploi conjonctif et de la grammaticalisation de la locution adverbiale au contraire.

Dans cette recherche, nous nous contenterons d'étudier le deuxième type de changement ${ }^{3}$. Cette enquête nous permettra de mesurer l'impact et les conséquences de ces changements sur le système grammatical, en commençant par le « micro-système $»^{4}$ des expressions adversatives pour arriver au « macro-système » c'est-à-dire le système grammatical même. Notre recherche s'appuie sur un corpus allant de l'AF jusqu'au XVII ${ }^{\mathrm{e}}$ siècle. Ce corpus est puisé dans trois bases de données différentes ${ }^{5}$ : la BFM, pour la période de l'AF, le DMF pour le moyen français (désormais MF) et Frantext à partir du XVI siècle. En plus de ce corpus numérisé, nous emprunterons parfois des exemples donnés par d'autres linguistes.

\section{L'hypothèse du remplacement de ains par au contraire est-elle soutenable?}

A les considérer d'un point de vue traditionnel, nous réalisons la différence des deux morphèmes ains et au contraire. Le premier appartient à la classe des conjonctions de coordination, il est toujours placé en tête du deuxième terme de la coordination. Le second est plutôt classé comme adverbe et se caractérise, par conséquent, par sa mobilité dans la proposition.

En ce qui concerne leur évolution, les deux mots ont subi des parcours très différents. La conjonction est d'origine latine, même si cette origine reste très discutée ${ }^{6}$. Elle proviendrait de antius, comparatif du latin vulgaire au sens de «plus tôt ». Dès les plus anciens textes français, ains parvient à fonctionner comme coordonnant adversatif après une proposition négative. En revanche, au contraire est une formation relativement récente et purement française. Elle ne parvient à fonctionner comme connecteur adversatif qu'à partir du $\mathrm{XVI}^{\mathrm{e}}$ siècle, même si elle apparaît bien avant. Pour la question du remplacement de ains, les études l'ont toujours résolue dans le cadre de son opposition à mais, ces études sous-entendent, quand elles ne le disent pas d'une manière explicite, que s'il y a eu remplacement c'est par cette dernière conjonction, vu que les deux conjonctions se sont confondues dans leur fonctionnement syntaxique (Rodriguez-Somolinos, 2000) et dans leur fonctionnement sémantique (Antoine, 1958-1962 : 1112-1157).

Pour notre part, nous avons éliminé cette hypothèse pour une raison toute simple que nous explicitons dans ce qui suit. Tous les linguistes qui ont opposé ces deux conjonctions (Melander, 1916; Antoine, 1958-1962 ; Kleiber, 1978; Rodriguez-Somolinos, 2000 et 2002) ont souligné la plus grande force adversative de ains. Par conséquent, les deux conjonctions ont toujours fonctionné en distribution 
complémentaire et jamais en distribution libre. Pour cette raison et étant convaincue que toute disparition est toujours compensée, nous proposons une autre hypothèse en dehors du cadre très restreint de l'opposition ains/mais. En effet, la disparition d'un morphème est toujours accompagnée soit de l'apparition d'un autre qui le remplace soit de plusieurs autres qui se partagent les différentes fonctions du morphème disparu. Selon Combettes (1995: 35), une perte sans remplacement n'est jamais attestée étant donné que les morphèmes disparus « peuvent toujours être 'traduits' assez fidèlement par une expression qui a survécu ou qui est de formation plus récente. »

Nous soutenons que s'il y a eu remplacement de ains, c'est par la locution adverbiale au contraire, même si les deux mots appartiennent à des catégories différentes du point de vue traditionnel. Mais d'un point de vue plus large, ils appartiennent à la catégorie des connecteurs. Nous affirmons qu'entre ces deux morphèmes, il y a beaucoup plus de points communs que de points de divergences. En effet, plusieurs linguistes $^{7}$ ont souligné la ressemblance frappante entre le fonctionnement de la conjonction ains et celui de la locution adverbiale moderne au contraire, mais sans parvenir à établir une relation entre la disparition de la première et l'apparition de la seconde. Ce sont ces points de rencontre entre le fonctionnement des deux termes qui feront l'objet de notre analyse, ensuite nous essayerons de retracer les étapes de l'évolution de la locution adverbiale qui se produisent en même temps que l'éclipse progressive du coordonnant, pour parvenir enfin à cerner les conséquences de ces deux changements sur le système grammatical.

\section{Les points de rencontre entre ains et au contraire}

Pour développer ce point, nous nous sommes appuyée surtout sur les travaux de Danjou-Flaux (1983 et 1986) pour la description de la locution adverbiale au contraire en français moderne (désormais FM), et ceux de Kleiber (1978) et Rodriguez-Somolinos (2000 et 2002) en ce qui concerne ains en ancien et moyen français.

La locution adverbiale moderne au contraire a un fonctionnement syntaxique et sémantique très riche. Pour décrire ce fonctionnement, Danjou-Flaux (1983 et 1986) recourt à une distinction entre un emploi monologal et un autre dialogal.

Pour notre part, nous n'allons pas faire une description détaillée du fonctionnement de ce connecteur, nous allons nous intéresser uniquement à un seul emploi de ce connecteur. Qu'il soit monologal ou dialogal, nous allons focaliser sur au contraire lorsqu'il oppose deux propositions complètes, il se trouve dans la configuration $p$ au contraire $q$, où $p$ et $q$ représentent des propositions complètes qui caractérisent obligatoirement le même fait. Cette précision faite, nous allons à présent confronter le fonctionnement syntaxique puis sémantique et enfin cohésif de la locution moderne au contraire, à celui de ains en ancien et moyen français.

Pour ce faire, nous avons opté pour une distinction entre l'emploi monologal et l'emploi dialogal de ces deux connecteurs, même si nous allons ramener à la fin ces deux emplois à une description unique.

\subsection{Ains/au contraire dans l'emploi monologal}

Dans l'emploi monologal, Danjou-Flaux (1983 et 1986) distingue trois fonctions différentes de $a u$ contraire, cette locution peut assurer une fonction substitutive, disjonctive ou antithétique. Seule la première fonction nous intéresse lorsque au contraire sert à introduire une proposition qui vient se substituer à la première proposition. Ce type d'emploi est syntaxiquement contraint, puisque le premier terme doit toujours être négatif, alors que le second est positif (Danjou-Flaux, $1983: 288$ et $1986: 106$ ).

(1) «En ce moment je n'ai pas envie de travailler; au contraire, je voudrais prendre des vacances ». (Exemple donné par Danjou-Flaux, $1983: 288$ ).

Cette caractéristique syntaxique concerne aussi ains et mais réfutatif; ces deux coordonnants ne peuvent être employés qu'après une négation syntaxique et très rarement après une négation lexicale en $\mathrm{AF}^{8}$. Pour 
au contraire, cette négation est le plus souvent syntaxique, mais elle peut très bien être lexicale. L'opposition que au contraire et ains opèrent n'est pas entre $p$ et $q$, mais elle est entre $p$ ' et $q$ ( $p$ ' étant l'énoncé positif correspondant à $p$. En d'autres termes $p=$ négation $+p$ ').

Ce premier trait syntaxique partagé entre l'un des emplois d'au contraire moderne et ains appelle un autre trait syntaxique inhérent au fonctionnement des deux connecteurs. Il s'agit de l'ordre des propositions reliées par ces deux morphèmes. Cet ordre n'est pas indifférent, il est toujours contraint, on ne peut avoir que $p$ au contraire/ains $q$, et jamais $q$ au contraire/ains $p$.

(1') *Je voudrais prendre des vacances, au contraire en ce moment, je n'ai pas envie de travailler.

Ceci vaut aussi pour ains. Voici un exemple cité par Kleiber :

(2) Mes les degrez mie n'avale, / Einçois s'arreste, ... (Chrétien de Troyes, Chevalier de la Charrette ou Lancelot, v.68-69)

(2’) *s'arreste, einçois les degrez mie n'avale. (exemple transformé par Kleiber, $1978: 280$ )

Si ains dans l'ancienne langue et au contraire dans l'usage moderne - et dans cet emploi précisément imposent obligatoirement l'ordre $\sim p$ ' ains/au contraire $q$, c'est parce que le contenu de chacun de ces deux connecteurs comporte ce que Kleiber appelle 'le trait rectificatif' pour ains et Danjou-Flaux 'le trait anaphorique' pour au contraire, et que nous appellerons 'le trait réfutatif' pour les deux. Dans les deux cas, le connecteur oblige à faire un retour en arrière afin de repérer le premier terme de l'opposition et de le rejeter tout en le remplaçant par une assertion plus adéquate. Les deux connecteurs effectuent "un double mouvement de rejet et d'assertion » (Danjou-Flaux, $1986: 103$ ), rejet de $p$ ' et assertion de son substitut.

Ces deux caractéristiques syntaxiques partagées par les deux connecteurs, à savoir la négation du premier terme et l'ordre contraint des propositions reliées par ains/au contraire, ne peuvent être sans conséquences sur le sens. En effet, la négation de $p$ est toujours polémique selon les termes de Ducrot (1984) dans ces deux types de construction, c'est-à-dire que le locuteur s'oppose à une assertion (réelle ou virtuelle) contraire et sous-jacente à l'énoncé négatif. En utilisant ains/au contraire q, le locuteur s'oppose à ce point de vue et le réfute, et $q$ vient se substituer à ce point de vue doublement rejeté, d'abord par la négation, ensuite par l'assertion de son substitut. Avec ains/au contraire, $p$ ' n'est mentionné que pour être annulé par la suite en lui substituant $q$, c'est-à-dire que $p$ ' n'est pas maintenu dans les propos du locuteur, il est considéré comme non valable. Avec mais réfutatif, par contre, « $q$ vient modifier, rectifier $p$ ' sans l'annuler, ce n'est pas une substitution mais une correction, une rectification, le locuteur oppose deux énonciations tout en présentant la deuxième comme préférable à la première » (Rodriguez-Somolinos, 2000 : 462).

Ce type de description permet de comprendre la plus grande force adversative toujours attribuée, par les linguistes, à ains par rapport à mais réfutatif ${ }^{9}$.

Cette opération qui permet d'annuler un terme de l'opposition en faveur d'un autre terme, qui est en fait son contraire ${ }^{10}$, présente les deux termes $[p$ ' et $q]$ de l'opposition comme des propositions incompatibles (les deux ne pouvant être vraies simultanément). Cette relation qui s'établit entre les deux propositions est à rattacher au connecteur qui fonctionne, selon Kleiber (1978), comme " opérateur d'inversion » pour ains, et comme «opérateur d'antonymie » pour au contraire selon Danjou-Flaux (1986). Les deux auteurs, même s'ils parlent de deux connecteurs différents, évoquent un même fonctionnement attribuable aussi bien à ains qu'à au contraire dans son emploi monologal substitutif. Ces deux connecteurs obligent à voir dans $p$ ' et $q$ des propositions inverses, même s'il n'y a aucune relation de contradiction entre elles. En d'autres termes, ains et au contraire ont cette capacité de transformer une simple différence en une contradiction radicale. Afin d'expliciter ce point, nous proposons un exemple cité par Kleiber (1978: 288) :

(3) Quant li escrit m'ot fet savoir / Que ce estoit trestot por voir / La fontaine au bel Narcisus, / Je me suis trez un poi ensus, / Que dedans n'ousai esgarder, / Ainz commençai a coarder, / Que de 
Narcisus me souvint / Cui malement en mesavint. (Guillaume de Lorris, entre 1225 et 1230, Roman de la Rose, 1509-1516)

Dans cet exemple, « ainz oblige à concevoir commencai a coarder comme le contraire de ousai esgarder» (Kleiber, 1978 : 289) d'ailleurs, Kleiber traduit ains par mais au contraire.

De même pour au contraire en FM, il peut soit opposer des contraires lexicaux (grand/petit, heureux/malheureux), soit «créer des contraires discursifs » selon les termes de Danjou-Flaux (1986: 107). Voici un exemple qu'elle propose :

(4) Cependant, lorsque les gentils bruits de pas se rapprochèrent de plus en plus, il eut honte d'effrayer la jeune fille. Au contraire, pour lui faire saisir qu'il était là, il siffla quelques passages de la chanson d'Ise qu'elle avait chantée tout à l'heure ${ }^{11}$. (Mishima, Le tumulte des flots, pp 71-72)

«Du point de vue de la langue, rien ne permet de considérer effrayer et siffler comme deux antonymes, car on peut siffler pour mettre en confiance, mais tout aussi bien pour provoquer la peur, le désarroi. Il s'agit donc d'une antonymie discursive, créée pour la circonstance » (Danjou-Flaux, 1986: 107-108). Autant dire que c'est « un opérateur d'inversion », pour reprendre le terme de Kleiber, tout comme ains.

Par ailleurs, au contraire substitutif, comme ains d'ailleurs, présente les deux propositions qu'il relie comme « occupant des positions situées aux antipodes l'une de l'autre » (Danjou-Flaux, 1986 : 106-107). La locution adverbiale creuse l'écart entre les deux termes de l'opposition. Ceci ne peut que rappeler la description que Rodriguez-Somolinos (2002 : 531) fait de ains et de son fonctionnement sémantique en ancien et moyen français. Ainsi, dans son emploi monologal, « ains présente $q$ comme incompatible avec $p$ ' et situe les deux propositions aux deux extrémités de l'échelle, il présente $p$ ' et $q$ comme radicalement opposés », et ceci indépendamment du contenu informatif des deux propositions.

Le rôle de l'ancien connecteur et de son homologue moderne est de souligner une opposition avec force qui provient justement de cette opposition radicale entre les deux termes, opposition qui peut être créée par le connecteur même. Ceci nous amène à parler d'un autre type de force inhérente à ces deux connecteurs. Il s'agit d'une force expressive signalée par Danjou-Flaux pour au contraire et par Rodriguez-Somolinos pour ains

Dans la suite, $p$ ains/au contraire (substitutif) $q$, le locuteur rejette le point de vue énoncé dans $p$ ' et prend en charge à la fois ce rejet - la négation étant polémique - et l'assertion de la justification de ce rejet par l'intermédiaire de $q$. dans ce type d'énoncé, le locuteur s'investit à fond dans ce qu'il dit. C'est ce qui a fait dire à Rodriguez-Somolinos (2000: 531-532) «tout comme au contraire en français moderne, au niveau expressif ains est un connecteur marqué subjectivement ».

Ajoutons enfin, que les deux connecteurs jouent un rôle important dans la cohésion du discours du locuteur et la succession de ses énoncés, puisque l'un comme l'autre oblige à mettre en rapport une proposition déjà énoncée et une autre à venir.

\subsection{Ains/au contraire dans le dialogue}

\section{- Après une assertion}

Le deuxième emploi caractéristique de ains en $\mathrm{AF}$, est celui qui consiste à rectifier, voire corriger, une assertion prononcée par un autre locuteur. Cet emploi rectificatif de ains est déjà partagé dès l'AF avec mais réfutatif. Dans cet emploi dialogal de ains, l'opposition se fait directement sur le point de vue positif énoncé par un énonciateur réel cette fois-ci. L'opposition se fait directement à $p$ '.

(5) - Il est d'Auchoirre / - Ains est franchois. (Courtois d'Arras, v. 207-208, exemple cité par Rodriguez-Somolinos, $2000: 463)$.

Ce même emploi est possible depuis le $\mathrm{XVI}^{\mathrm{e}}$ siècle, mais avec au contraire dialogal qui rejette et vient remplacer une assertion précédente d'un autre locuteur. Dans ce cas-là, la négation dans $p$ ' n'est pas 
obligatoire dans la mesure où ains/au contraire comportent implicitement cette négation de $p^{\prime}$. Voici un exemple avec au contraire :

(6)- Comment faut-il prévenir les disettes et les famines ? / - Rien de plus simple. Il faut défendre l'exportation des grains. / - Au contraire, il faut accorder une prime à ceux qui les exportent. (Joseph de Maistre, 1810, Des constitutions politiques et des autres institutions, p. 4-5, Frantext)

Les deux exemples correspondent au même schéma :

$$
\begin{aligned}
& \mathrm{A}-p \\
& \mathrm{~B} \text { - ains / au contraire } q
\end{aligned}
$$

Dans un monologue, nous pouvons obtenir les gloses suivantes :

$\Rightarrow$ «Il n'est pas d'Auchoirre ains est franchois »

$\Rightarrow$ «Il ne faut pas défendre l'exportation des grains, au contraire, il faut [au contraire] accorder une prime à ceux qui les exportent $»$.

La négation dans le monologue montre bien que $p$ rapporte un discours d'un énonciateur virtuel. L'emploi monologal et dialogal sont ainsi ramenés à une même description.

Au contraire a donc remplacé aussi bien ains rectificatif que mais réfutatif, employés dans le dialogue et figurant après une assertion lorsque l'opposition porte directement sur cette assertion. Il les a remplacés, puisque l'un est sorti de l'usage et l'autre (mais réfutatif) est devenu impossible dans ce type d'emploi en FM. En effet, selon Anscombre et Ducrot (1977 : 39), mais réfutatif doit obligatoirement faire «l'objet d'un acte de parole unique ».

\section{- Après une interrogation}

La même opposition peut être exprimée après une interrogation ${ }^{12}$. Dans ce cas-là, l'opposition ne se fait pas à l'acte de l'interrogation même, mais à la réponse positive éventuelle.

(7) « Sire, entre moi Gaheriet vions en la forest; vendroiz vos ? / - Nenil, fet Lancelot, ainçois remeindrai, car ge ne sui ore mie bien aisiez d'aller a ma volenté ». (Anonyme, 1230, Mort le Roi Artu, p. 114, BFM)

Avec au contraire :

(8) - Dites-moi, monseigneur, vous enorgueillissez-vous d'inspirer un semblable mouvement ? / - Au contraire, j'en suis humilié, et surtout affligé. (Mme de Genlis, 1782, Adèle et Théodore, p. 103 , lettre 16, Frantext)

De l'emploi dialogal réfutatif de ains et d'au contraire découle ce que l'on appelle, l'emploi 'rectificatif'. Dans ce cas, l'opposition est métalinguistique, elle porte sur un terme énoncé par le locuteur 1, auquel le locuteur 2 s'oppose, alors il le remplace par un terme plus adéquat et souvent plus fort. Voici un exemple avec ains :

(9) Estes vos, dist Tourgis, blechies ? / - Blechies ? dist il, ains sui tues. (Anonyme, à partir de 1180, Roman de Renart, XV, v. 491, exemple donné par Antoine, 1958-62:1127)

Voici un autre avec au contraire :

(10) - (...) Cette cicatrice que vous m'avez faites au dessous de l'œil, et que ne devrais vous pardonner de ma vie, n'a pas peu contribué à m'enlever ce dernier, ce frivole avantage. / Sérieux, au contraire aux yeux des femmes mais frivole à ceux des hommes. (G. Sand, 1843, Consuelo, p. 156, Frantext)

La correction porte dans les deux cas sur un terme énoncé par le premier locuteur, à savoir "blechies" avec ains et "frivole" avec au contraire. Ces deux termes sont jugés inadéquats et méritent donc correction selon le deuxième locuteur. Dans cet emploi l'opposition se fait aussi à une énonciation effective puisqu'elle se réalise dans le cadre d'un dialogue. 
Pour une description unique des deux connecteurs, nous pouvons dire qu'ils ont la propriété de pouvoir toujours s'opposer à une énonciation positive $p$ ' attribuée à un locuteur réel ou virtuel que ce soit dans le monologue ou dans le dialogue.

Un dernier point commun unit les deux connecteurs : ains, comme au contraire, ne peut porter que sur une assertion, il n'est jamais employé derrière un ordre, et derrière une interrogation, les contraintes comme nous l'avons mentionné plus haut sont très fortes. La raison est toute simple, les deux connecteurs agissent avec force afin d'annuler ce qui précède, et un ordre lorsqu'il est proféré, peut être contesté mais non annulé. Après une interrogation, ains/au contraire n'annulent pas l'acte de parole mais une réponse éventuelle.

La locution adversative moderne, au contraire, s'est donc frayé un chemin parmi les expressions adversatives et réfutatives, elle a accaparé l'emploi le plus original du coordonnant ains, elle en a hérité la force adversative, lui permettant de transformer une simple différence en une opposition radicale. Par ailleurs, elle a pris aussi l'emploi dialogal aussi bien de ains que celui de mais réfutatif de l'ancien et du moyen français.

\section{La grammaticalisation de au contraire et le déclin de ains}

\subsection{Au contraire : un parcours de grammaticalisation ${ }^{13}$}

Il est pratiquement incontestable de reconnaître, à présent, qu'entre la première étape d'une grammaticalisation et l'étape finale où le mot intègre le système grammatical, il existe une ou des étapes transitoires de variation où l'ancienne et la nouvelle valeur du mot en mutation coexistent. C'est ce qui semble correspondre à l'évolution de au contraire. Par ailleurs, le contexte dans lequel le mot apparaît semble pouvoir déterminer l'étape à laquelle en est l'évolution du nouveau morphème. Ce contexte joue un rôle capital dans tout cas de grammaticalisation selon Heine (2002). Afin de décrire l'évolution de cette locution adverbiale, nous allons procéder par période tout en mettant l'accent sur les deux premières étapes. Nous allons, par ailleurs, faire appel à la typologie des textes dans lesquels le syntagme apparaît, lorsque ses emplois vont devenir de plus en plus variés, notamment au XIV ${ }^{\mathrm{e}}$ siècle $^{14}$. De fait, au contraire paraît à première vue une expression qui n'a pas subi de véritable changement sémantique, étant formée à partir de l'adjectif substantivé contraire, lui-même exprimant, depuis le latin, une opposition. Cependant, ce syntagme prépositionnel est, à notre avis, l'exemple type qui permet de voir clairement les étapes de son évolution. En FM, c'est une locution adverbiale formée de au et de l'adjectif substantivé contraire. Au est la forme contractée de a le qui a donné al jusqu'au milieu du XI ${ }^{\mathrm{e}}$ siècle, selon le Robert Historique, remplacée par au à partir du XIII ${ }^{\mathrm{e}}$ siècle. Quant au syntagme nominal le contraire, il est le résultat de la substantivation de l'adjectif contraire. Cet adjectif est d'origine latine, il a pour étymon contrarius signifiant «en face de, du côté opposé ». Il est dérivé de contra, préposition latine de sens d'abord spatial «en face de, vis-à-vis », qui a donné la préposition moderne contre et a contribué à la formation de plusieurs mots. Si la préposition contre continue à avoir, en FM, entre autres, une valeur spatiale, l'adjectif contraire n'a gardé qu'un sens oppositif, dérivé d'un sens spatial à l'origine, comme nous allons essayer de le démontrer dans notre analyse.

Dans notre corpus, nous avons repéré la première occurrence de l'adjectif substantivé dans l'œuvre anonyme Chanson de Roland qui a été composée vers 1100 (v.290, BFM)

(11) Ço set hom ben que jo sui tis parastres, / Si as juget qu'a Marsiliun en alge. / Se Deus ço dunet que jo de la repaire, / Jo t'en muvra une si grant contraire / Ki durerat a trestut tun edage.

L'adjectif peut être désormais employé comme nom avec (ou sans) déterminant. Il est tantôt masculin, tantôt féminin. Pour ce qui est du syntagme prépositionnel au contraire, il apparaît pour la première fois dans notre corpus vers 1283, chez Philippe de Beaumanoir, dans Coutumes de Beauvaisis, volume 1. Avant cette date, on rencontre l'expression a contraire qui est, à notre avis, l'ancienne forme de la locution moderne. Ce syntagme commence à être utilisé à partir du XII ${ }^{\mathrm{e}}$ siècle. La présence de l'article n'étant pas obligatoire, le syntagme a pris cette forme qui ne comporte que la préposition et le substantif. 
Nous avons dénombré 23 occurrences de ce syntagme, dans les textes d'AF, numérisés par la BFM. Il apparaît dans des contextes peu variés. En effet, a contraire apparaît le plus souvent (13 fois sur 23) derrière un verbe de mouvement, tels que torner, retorner ou venir. Dans cette configuration, le substantif est souvent séparé de la préposition par l'adjectif grant; les deux éléments du syntagme jouissent encore de cette liberté permettant au substantif d'être déterminé par un adjectif, en d'autre termes la relation entre les deux éléments est encore lâche. Le syntagme a tout l'air de fonctionner dans un contexte qu'on pourrait qualifier de figé et qui trouve donc son emploi dans le cadre de locutions verbales formées à partir d'un verbe de mouvement, avec une préférence notable pour le verbe torner (11 fois sur 13), et du syntagme a contraire. Après ces verbes de mouvement, on s'attend à ce que le sens soit spatial et dynamique, et que la locution signifie avec le verbe de mouvement qui l'accompagne «tourner du côté opposé » : il n'en est rien. Elle est toujours -du moins dans notre corpus- employée dans un sens figuré. Par métaphorisation, ces prédicats passent d'un sens spatial référentiel (attesté en AF pour le substantif contraire) à un sens plus abstrait et signifient alors «contrairement à ce qu'on attend ou faire quelque chose en retour ou en représailles », comme l'illustrent les exemples suivants :

(12) Poines, travalz et granz dolors, / Molt me dotai de cel ostage / Que vos eüstes an Cartage, / N'an perdissiez tot vostre afaire / Ou vos tornast a grant contraire. (Anonyme, vers 1155, Eneas, tome 1, v. 2856, BFM)

(13) Car tuit autre mal sont amer / Fors seulemant celui d'amer ; / Mes cil retorne s'amertume en dolçor / Et an soatume et sovant retorne a contraire. / Mes cele qui bien sot l'afaire li respont : / " Ja ne dotez rien, de vostre mal / Vos dirai bien la nature et le non ansanble. (Chrétien de Troyes, 1176, Cligès, v. 3065, BFM)

Le syntagme a probablement fonctionné avant de passer au sens figuré comme locatif après ces verbes de mouvement. Dans les exemples que nous avons proposés, il se comporte comme complément circonstanciel de manière lié au verbe et il agit au niveau propositionnel avec une fonction essentielle.

Quant à l'emploi de la préposition $\grave{a}$, il s'explique ${ }^{15}$ par le fait que sa combinaison avec un verbe de mouvement tel que torner, retorner ou venir et le substantif contraire, au sens de " du côté opposé », ne peut lui donner qu'une valeur spatiale au départ, celle de la préposition vers du FM, et toute la locution verbale signifiait « faire un mouvement vers le côté opposé » au sens direct ou figuré ${ }^{16}$. La préposition est requise par le verbe même et, à notre avis, c'est elle aussi qui va permettre l'élargissement des contextes dans lesquels le syntagme peut apparaître, étant donné que cette préposition a une valeur «non marquée » comme le dit Combettes (2003: 278) et peut se colorer de diverses nuances sémantiques. C'est justement cette préposition qui a permis le deuxième emploi de a contraire, ce dernier apparaît dès le XII ${ }^{\mathrm{e}}$ siècle dans notre corpus, il s'agit de son emploi derrière le verbe être. Le syntagme dépend d'un verbe d'état et non de mouvement, on passe des lieux opposés aux états opposés sans forcément passer par un contexte explicitant la valeur spatiale originelle du substantif. Il agit toujours dans le cadre propositionnel, puisqu'il fonctionne comme attribut du sujet. Dans cette configuration, la locution verbale signifie « être contrarié » ou « s'opposer à quelque chose ou à quelqu'un », comme l'illustrent les exemples suivants :

(14) Icest' ovre m'est a contraire. (Thomas, entre 1172 et 1176, Tristan, v. 464, BFM)

(15) Bien li conmande l'emperere qu'il soit et sire et comandere et ne li soit nus a contraire de quanques onques valra faire. (Gautier d'Arras, vers 1176-1184, Eracle, v. 1917, BFM)

Ce sont les deux seuls contextes dans lesquels a contraire se rencontre durant les XII ${ }^{\mathrm{e}}$ et XIII ${ }^{\mathrm{e}}$ siècles.

Ce n'est que vers la fin du XIII ${ }^{\mathrm{e}}$ siècle, que le syntagme au contraire commence à être employé d'une manière de plus en plus régulière, écartant ainsi le syntagme dépourvu de l'article. D'ailleurs nous n'avons relevé que 4 occurrences de ce dernier syntagme au XIV siècle, 10 au XV et aucune au siècle suivant. Le syntagme ayant acquis le sens de l'opposition après le verbe être, il va pouvoir étendre ses emplois à d'autres types de verbes, emplois auparavant impossibles. En effet chez Beaumanoir, au contraire est employé après le verbe faire avec le sens d' " agir d'une manière différente ou contraire » :

(16) (...) ne nus usages que l'en ait fet au contraire ne doit valoir, car usage qui est fes contre le commun pourfit ne doit pas valoir que la chose ne soit ramenee a son ancien estat. (Philippe de Beaumanoir, vers 1283, Coutumes de Beauvaisis, volume1, p.370, BFM)

Chez Joinville, au début du XIV ${ }^{\mathrm{e}}$ siècle, au contraire figure après le verbe dire dans le cadre d'une incise, nous reviendrons sur cet emploi un peu plus loin. 
Dans tous les cas étudiés plus haut, et durant toute la période de l'AF, le syntagme agit au niveau propositionnel et permet de dénoter une manière de réalisation du procès.

C'est à partir du XIV ${ }^{\mathrm{e}}$ siècle qu'une extension des emplois et des valeurs de au contraire apparait, elle va aboutir à une re-catégorisation du syntagme en tant que locution adverbiale. Nous pensons même que la période du MF représente l'étape transitoire entre le début et l'achèvement du parcours de grammaticalisation de la locution. Le syntagme va apparaître dans des contextes tout à fait nouveaux tout en continuant à fonctionner dans le cadre des contextes précédemment étudiés avec a contraire. Cette période représente pour la locution ce que Marchello-Nizia (2006: 22) appelle «une synchronie complexe ou hétérogène » qui marque la coexistence de deux systèmes. C'est ce que nous allons tenter de prouver. $\mathrm{Au}$ cours des $\mathrm{XIV}^{\mathrm{e}}$ et $\mathrm{XV}^{\mathrm{e}}$ siècles, au contraire continue à se rencontrer dans les mêmes configurations décrites plus haut, avec cependant une extension à d'autres verbes, qu'ils soient des verbes de mouvement ou des verbes factitifs, pour fonctionner toujours comme complément circonstanciel de manière intraprédicatif. En effet, à partir du $\mathrm{XIV}^{\mathrm{e}}$ siècle, d'autres verbes de mouvement deviennent possibles avant au contraire. Nous pouvons citer à titre indicatif les verbes aller, traire, mener, muer, mouvoir, etc., ce sont des constructions dans lesquelles au contraire peut entrer et qui témoigneront jusqu'au $\mathrm{XVI}^{\mathrm{e}}$ siècle de l'origine spatiale d'au contraire. En voici deux exemples :

(17) Et sachiez que s'il eüst osé en nulle maniere, il en eüst prins vengence; mais il estoit si fort lié et l' avoit tellement promiz que nullement il n'eüst osé aler au contraire de ce qu'il avoit promiz a Gieffroy. (Anonyme, 1350, Berinus, T. 2, p. 57, DMF)

(18) Mais concupiscence maine et trait au contraire, car concupiscence peut mouvoir chascune des parties de l'ame. (Nicole Oresme, 1370, le Livre de ethiques d'Aristote, p. 374, DMF)

La dimension spatiale de la construction n'est pas perdue, d'autant plus que l'expression "le vent au contraire" est assez récurrente dans le corpus du DMF, il s'agit du vent venant du côté opposé :

(19) Et ilz ne s'en bougeront ja pour le chien / Tout seul, especialement car ilz aront / Le vent au contraire, qu'ilz n'orront / Ni sentiront rien ne des chienz ne des genz. (Gaston Phebus, 1387, Livre de chasse, p. 259, DMF)

Par ailleurs, des verbes comme proceder et traiter enrichissent la palette des verbes factitifs, celle-ci ne comportait auparavant que le verbe faire.

(20) Gramaire est necessaire aux enfans, joieuse aux anchiens, doulche compaigne des choses secrettes, en laquelle de toutes estudes a plus d'euvre et mains de monstre. Combien que pluseurs par orgueil veullent commenchier a apprendre les sciences qui sieuent, qui se deçoivent par ainssi proceder au contraire. (Jean Daudin, 1360, De La Erudition, p. 77-78, DMF)

(21) Et discernons des ore mais / Tous ceulx pour excommenïez / Et, se mestier est, aggregiez, / Qui le traiteront au contraire. / Congié lui donnons de contraire / Nouvel marïage et plus hault. (Estoire de Griseldis en rimes et par personnage, 1395, v. 1970, BFM)

Avec le verbe être, la structure est toujours employée, mais elle peut avoir une nouvelle signification en plus de l'ancienne, elle est souvent équivalente à « à l'inverse » :

(22) Car celui qui peut et a du bien plus que selon ceste proportionnalité, il fait injustice. Et celui qui en moins, il suefre injustice. Et en mal et poinne, labeur ou damage, il est au contraire. (Nicole Oresme, 1370, le Livre de ethiques d'Aristote, p. 287, DMF)

On voit même l'expression au contraire de se développer après le verbe être, elle s'apparente à une locution prépositive qui signifie « être opposé à quelque chose ou à quelqu'un »

(23) De ce point nous faites scillence, / Evesque et si vous vueilliez taire, / Car si plus estes au contraire / De Pierre Lyon, je vous jur / Que je mesme, soiez en seur, / De ma terre vous chaceray / Et de l'eveschié vous toldray. (Anonyme, 1347, Miracle de saint Guillaume du Desert, p. 9, DMF) Tout ce que nous venons de décrire concerne l'élargissement des mêmes emplois antérieurs sans véritable changement ni du contexte ni des valeurs. Cependant, des modifications importantes des contextes dans lesquels au contraire se rencontre sont visibles dès le début du XIV ${ }^{\mathrm{e}}$ siècle. En effet, l'expression pouvant exprimer une opposition a été récupérée dans le domaine juridique. Dans ce type de texte au contraire va 
même acquérir le sens de l'opposition d'une manière générale et va désigner l'étape de l'opposition dans un procès avec généralement un verbe du dire tel que dire, repliquer, nier, etc. :

(24) Et au contraire, ledit Mahiet, ou nom que dessus, eust nyee la demande et la saisine devant dite. (Chartes de l'abbaye de Saint-Magloire, tome3, 1330, p. 522, DMF)

(25) Le procureur du Roy propose et dit au contraire que Michau Laillier et J. Tarenne, qui sont marchans notables, ont prins ledit pont de St Michiel pour edifier chascun un costé à ZZZXXXIJ libvres à ZZZIJ vies seulement, et si baillent mil libvres à parfaire le pont, et ne reste que à bailler le decret, à quoy partie s'oppose en offrant ZZZVIIJ libvres de rente seulement que baille. (Nicolas de Baye, 1400, Journal, T 1, p. 255-256)

Le syntagme désigne une étape du procès, celle qui permet à l'une des deux parties de présenter des contre-arguments afin de réfuter ce qui a été dit auparavant. C'est une étape qui survient obligatoirement après une autre. La locution est souvent placée en tête de l'énoncé, puisqu'elle enchaîne forcément sur un dire précédent et présente un dire opposé. Si sur le plan syntaxique, au contraire, est toujours complément circonstanciel qu'on pourrait qualifier de temporel et qui sert à dater le procès du verbe, sur le plan sémantique, il acquiert déjà un sens de réfutation d'une énonciation précédente. Sans qu'il ne se charge lui-même de cette réfutation, il en souligne le moment.

Ce type d'emploi dans les textes juridique provient, en fait, d'un autre emploi encore plus ancien et que nous avons évoqué sans le traiter. Il s'agit de l'emploi de la locution derrière le verbe dire dans le cadre d'une incise chez Joinville.

(26) « (...) car peudomme est si grand-chose et si bone chose que neis au nommer emplist il la bouche. » Au contraire disoit il que male chose estoit de prendre de l'autrui, car le rendre estoit si grief que neis au nommer le rendre escorchoit la gorge... (Jean de Joinville, entre 1305 et 1309, Mémoire ou Vie de Saint Louis, p. 16, BFM)

La nouveauté dans cet emploi peut être décrite à plusieurs niveaux. Elle concerne d'abord le type du verbe jusque-là impossible avec au contraire et des structures comme parler, dire, arguer au contraire vont devenir de plus en plus usitées au XIV ${ }^{\mathrm{e}}$ siècle. Le changement concerne ensuite la syntaxe du syntagme, il peut désormais être postposé au verbe et souvent placé en tête de la proposition. Cette dernière position lui permet, s'il se trouve entre deux énonciations différentes, de les opposer soit sur le plan de la forme soit sur le plan du contenu. A partir de ce moment le syntagme va commencer à fonctionner comme locution adverbiale dont les éléments deviennent indissociables. Et faisant preuve de cohésion, le syntagme va pouvoir être intensifié, dans tous ses emplois, par l'adverbe tout et plus rarement par l'adverbe fort :

(27) Or puet advenir aprés sa mort moult de transmutacions en ses filz ou neveus et puet estre que aucune fois soit bons et riches et en grans honneurs et en grans dignités, et aucunes fois avient tout au contraire. (Nicole Oresme, 1370, Le Livre de ethiques d'Aristote, p. 131, DMF)

Cet emploi après le verbe dire va se développer au $\mathrm{XIV}^{\mathrm{e}}$ siècle, en particulier dans les textes scholastiques. En effet, nous avons relevé chez Daudin dans De la Erudition édité en 1360, 23 fois sur 47 cas où la locution est employée dans une incise toujours avec le verbe dire, elle sert à opposer, souvent sur le plan de la formulation, deux énonciations différentes. Les deux énonciations reliées par au contraire comportent souvent des termes antithétiques :

(28) Pour che dist l'Apostre aux Galathiens ou ..\%VIe.. chapitre : «Instruiséz chelui qui aprend en esperit de bonne doulceur». Au contraire est dit en Proverbes ou ..\%XIXe.. chapitre : «L'esperit qui se corrouche de legier, qui le pourra soubstenir ?». (Jean Daudin, 1360, De La Erudition, p. 189, DMF)

(29) De rechief moult vault a doctrine bien scavoir parler, dont dist Therence : «Rien n'est qui ne puist estre empirié par mal prononcier». Au contraire dist Tulle : «Il n'est rien si horrible, ne si mal ordonné, qui ne resplendisse quant il est bien prononchié». La parole doit estre simple, car comme dist Juvenal en son quart livre : «Habondance de paroles nuiseuses par beau parler samblent savoureuses». (Jean Daudin, 1360, De La Erudition, p. 17-18, DMF) 
Même si les deux énonciations vont dans le même sens, c'est-à-dire que l'une ne réfute pas l'autre, elles s'opposent, tout de même, puisque chacune présente le phénomène en question selon un point de vue différent. Le vocabulaire employé ne fait qu'accentuer cette opposition : (28) esperit de bonne doulceur/ L'esperit qui se corrouche de legier ; (29) mal prononcier/ bien prononchié.

Dans ces mêmes types de textes en plus des textes philosophiques, un autre emploi fait son apparition avant de se généraliser dans les autres types d'écrit, c'est celui qui est illustré par les deux exemples suivants :

(30) Clareté en la face,doulceur en paroles coulourent moult obedienche du serviteur. $A u$ contraire composition obscure du corps et face tristre, noircie de tenebres, signifient devotion ou joieusseté estre separee du corage. (Daudin, J., 1360, De La Erudition, p. 240, DMF)

(31) Mais quant il [les fols hardis] sont es perilz et ou fait, ilz defaillent. Mais au contraire, les fors es operacions et fais perilleus sont aguz et aspres, constans et perseverans. Et au devant il sont paisibles senz estre esmeüs. (Nicole Oresme, 1370, Le Livre de ethiques d'Aristote, p. 131, DMF)

Cet emploi n'est pas différent du précédent dans la mesure où on retrouve la même opposition du vocabulaire. De plus, au contraire relie deux énonciations appartenant au même locuteur et non à deux locuteurs différents. C'est d'ailleurs ce qui explique l'absence du verbe dire. Cette contrainte s'est donc relâchée et c'est ce qui va permettre à la nouvelle locution d'opposer deux propositions dans le cadre d'une énonciation unique pour signifier «par contre» ou « inversement». C'est ainsi que la locution quitte le champ de la proposition, de par sa position initiale dans la phrase, pour participer à la cohésion textuelle en mettant en rapport deux propositions. Elle intègre ainsi le domaine textuel en tant qu'adverbe de liaison.

$\mathrm{Au} \mathrm{XV}^{\mathrm{e}}$ siècle, ces deux derniers emplois vont devenir possibles dans tous les types d'écrit y compris dans les textes juridiques :

(32) Et, eulz assemblez ainsi que dit est, survindrent les procureur et advocat du Roy, disans que nouvellement ilz avoient parlé au duc de Bedford, regent, et au chancelier de France de l'estat et de l'entretenement de la Court et du paiement des gaiges d'icelle Court, en leur remonstrant l'onneur et le grant bien qui povoit avenir en l'entretenement de la Court, et, au contraire, les esclandes et inconveniens. (Clément de Fauquembergue, 1431, Journal, T. 3, p. 81, DMF)

L'ellipse du verbe de déclaration remontrer avec au contraire est bien claire dans le cadre d'une énonciation appartenant à un seul locuteur.

C'est seulement à partir du $\mathrm{XV}^{\mathrm{e}}$ siècle que l'opposition exprimée par la locution va devenir une véritable réfutation de l'énonciation qui précède, toujours via un contexte particulier. Déjà implicite à la locution dans les textes juridiques, la réfutation va devenir explicite et saillante lorsque au contraire va commencer à appuyer ains et mais réfutatif :

(33) Je ne le dis, et ce scet Dieux, pour toy flater ne blandir, ne donner matiere d'avoir vaine gloire, ains tout au contraire. (Christine de Pizan, 1416, Epistre de la prison de vie humaine, p.37, DMF)

Dans cet emploi, au contraire a permis à ains d'être complété uniquement par cette locution et non par une proposition positive qui rejette la proposition précédente. Au contraire passe ainsi à une autre étape de son évolution, il est désormais capable d'être employé d'une manière absolue c'est-à-dire sans qu'il ne soit complété par une proposition, et ce afin de véhiculer la réfutation d'une énonciation précédente. Avec ce type d'emploi le locuteur s'investit dans son dire en prenant en charge aussi bien le rejet de l'énonciation précédente que l'affirmation de son contraire.

Cependant, cette réfutation opérée par au contraire va rester marginale tout au long du $\mathrm{XV}^{\mathrm{e}}$ siècle, ce sont les autres emplois et valeurs qui sont les plus représentés dans notre corpus.

En effet, dans les textes dépouillés du $\mathrm{XV}^{\mathrm{e}}$ siècle, nous avons dénombré 11 fois mais au contraire et une seule fois ains au contraire sur un total de 215 occurrences. Au XVI ${ }^{\mathrm{e}}$ siècle, par contre, ces compositions 
vont devenir très courantes, comme on le verra un peu plus loin, et on va perdre les autres emplois d'au contraire pour n'en garder que le sens moderne. La locution va même étendre ses emplois oppositifs, elle va pouvoir figurer dans le dialogue pour réfuter une énonciation d'un autre locuteur. Ce qui prouve qu'elle appuie encore ses capacités énonciatives et pragmatiques pour participer à l'expression de la subjectivité du locuteur et son investissement dans son énoncé.

Le parcours de grammaticalisation de cette locution semble ainsi répondre aux schémas récurrents de la grammaticalisation : Dépendance propositionnelle $>$ Cohésion textuelle $>$ cohérence énonciative.

Lieu $>$ manière $>$ opposition

Pour finir voici le tableau illustrant l'évolution de l'emploi de au contraire :

Tableau1 : évolution des emplois de au contraire depuis

le XII ${ }^{\mathrm{e}}$ siècle jusqu'au XVII ${ }^{\mathrm{e}}$ siècle

\begin{tabular}{|c|c|c|c|c|}
\hline Période & $\begin{array}{c}\text { Nombre de textes } \\
\text { dépouillés }\end{array}$ & a contraire & au contraire & Source \\
\hline XII $^{\mathrm{e}}$, XIII ${ }^{\mathrm{e}}$ et début du XIV & 68 & 23 & 3 & BFM \\
\hline De 1330 à 1399 & 91 & 4 & 246 & DMF \\
\hline De 1400 à 1499 & 123 & 10 & 215 & DMF \\
\hline De 1500 à 1599 & 148 & 0 & 789 & Frantext \\
\hline De 1600 à 1699 & 571 & 0 & 2653 & Frantext \\
\hline
\end{tabular}

\subsection{Le déclin de ains}

Pour exprimer une opposition après une proposition négative, l'AF se servait de ains et plus rarement, selon Rodriguez-Somolinos (2000: 457), de mais réfutatif. "Ce n'est qu'à partir du XIV siècle que l'usage de mais2 augmente, au détriment de ainz »C'est justement à partir de ce siècle que commence la décadence du connecteur réfutatif, pour finir par être très peu employé au $\mathrm{XVI}^{\mathrm{e}}$ siècle comme l'illustre le tableau suivant :

Tableau2 : emplois de ains/ainçois depuis

le XII 's siècle jusqu'à leur disparition

\begin{tabular}{|c|c|c|c|}
\hline Siècle & $\begin{array}{c}\text { Nombre de textes } \\
\text { dépouillés }\end{array}$ & $\begin{array}{c}\text { ainz }{ }^{17} / \text { Ainçois toutes } \\
\text { formes confondues }\end{array}$ & Source \\
\hline XII $^{\mathbf{e}}$ & 29 & 1338 & BFM \\
\hline $\mathbf{X I I I}^{\mathbf{e}}$ & 36 & 1862 & BFM \\
\hline $\mathbf{X I V}^{\mathbf{e}}$ & 91 & 1191 & DMF \\
\hline $\mathbf{X V}^{\mathbf{e}}$ & 123 & 840 & DMF \\
\hline $\mathbf{X V I}^{\mathbf{e}}$ & 148 & 1203 & Frantext \\
\hline $\mathbf{X V I I}^{\mathbf{e}}$ & 571 & 1450 & Frantext \\
\hline $\mathbf{X V I I I}^{\mathbf{e}}$ & 563 & $4^{18}$ & Frantext \\
\hline
\end{tabular}

$C^{\prime}$ 'est donc au moment où au contraire commence à étendre ses emplois oppositifs que ains commence à perdre du terrain jusqu'à ce qu'il tombe en désuétude au XVII ${ }^{\mathrm{e}}$ siècle. 


\section{Ains/au contraire de la cooccurrence à la concurrence}

A partir du $\mathrm{XV}^{\mathrm{e}}$ siècle, on a déjà commencé à appuyer ains et mais réfutatif par la nouvelle locution au contraire. Dans le corpus du XV $\mathrm{XV}^{\mathrm{e}}$ siècle, nous n'avons repéré qu'une seule occurrence ains au contraire chez Christine de Pizan et que nous avons citée plus haut. C'est au XVI siècle que cet emploi va se répandre et devenir assez courant. En voici un exemple de Théodore de Bèze (1583, Chrestiennes médiations Médiation sur li psalme, p. 78, Frantext) :

(34) (...) t'offrant cependant cela mesme que tu m'as donné, et qui je sçay que ta grace tu ne rejettes point ains au contraire tu recois comme tant agréable, que mesme sans cela tout ce service exterieur te desplait...

$\mathrm{Au} \mathrm{XVII}^{\mathrm{e}}$ siècle, cet emploi est senti comme redondant et, par conséquent, blâmé par certains remarqueurs du siècle. Vaugelas $(1650: 151)$ dit dans ses Remarques sur la langue françoise à propos de cette expression : "On dit maintefois à la Cour en raillant, et de la même façon que l'on dit ains au contraire ».

Cependant, c'est à partir du $\mathrm{XVI}^{\mathrm{e}}$ siècle que la nouvelle locution oppositive va commencer à relayer l'ancienne conjonction déjà en net recul par rapport au MF. Au contraire en expansion peut désormais être employé pour opposer deux propositions caractérisant un même fait, dont la première qui est négative, est réfutée par une seconde positive :

(35) « Mais il ne prit pas son chemin vers Amurat comme il disoit, au contraire il tourna court tout aussi tost qu'il fut hors des trenches, pour aller gaigner le Danube ». (Blaise de Vigenière, 1577, L'Histoire de la décdence de l'Empire grec, et establissement de celuy des Turcs, comprise en dix livres par Nicolas Chalcondyle [trad.], p. 486, Frantext)

Cet emploi d'au contraire va se répandre au XVII ${ }^{\mathrm{e}}$ siècle $\left(78\right.$ occurrences au $\mathrm{XVI}^{\mathrm{e}}$ siècle de au contraire après négation, et $2341 \mathrm{au} \mathrm{XVII})^{\mathrm{e}}{ }^{19}$ :

(36) « Je ne suis pas de ceux qui l'ont accusé d'avoir tué Cléandre, au contraire je suis celle qu'ay defendu son innocence, et pour l'amour de luy celle de sa dame ». (Vital d'Audiguier, 1624, Histoire trage-comique de nostre temps, sous les noms de Lysandre et de Caliste, p. 470, Frantext).

\section{L'élection de au contraire ou l'autorégulation du système}

Au contraire présente plusieurs avantages par rapport à ains. Dans l'opposition, ains exprimait soit la réfutation forte, et c'est son emploi le plus fréquent depuis l'AF jusqu'à sa sortie de l'usage au XVII ${ }^{\mathrm{e}}$ siècle, soit la rectification dialogale ou monologale. Son sémantisme est affecté par une syntaxe très contrainte (le coordonnant ne peut être employé qu'après une négation). Pour ce qui est de la locution au contraire, elle va, comme nous espérons l'avoir prouvé, remplacer ains dans ces deux emplois, mais elle va aussi développer un fonctionnement oppositif très riche. Cette locution peut avoir un emploi relatif ou absolu. Ce deuxième emploi était impossible avec ains. De plus, la contrainte syntaxique de la négation n'affecte qu'un seul des emplois de la locution.

Par ailleurs, cette locution a l'avantage, par rapport à ains, d'être une expression uniquement oppositive, elle ne peut exprimer que l'opposition même si elle a développé un champ d'action assez large dans l'expression de l'opposition ${ }^{20}$. Ains, par contre, est resté un mot polyvalent jusqu'au XVI $\mathrm{Xiècle}^{21}$. C'est ce qui fait dire à Antoine (1962: 1140) que «Bien entendu, des mobiles tenant plus étroitement au système de la langue ont agi de leur côté : ains outil de coordination n'est pas mort seul, mais en même temps que la locution subordonnante ains que, et la préposition ains (encore employée par Marot) ». C'est en note qu'Antoine souligne cette disparition de ains conjonction de coordination, préposition et locution conjonctive. En effet, pour notre part, nous croyons que la polyvalence de ains est l'une des raisons de sa dégénérescence. La disparition de cette forme, comme l'a bien vu Antoine, peut être expliquée par une cause interne au système, qu'on pourrait définir en terme de tendance à l'isomorphisme ${ }^{22}$. A partir de la description de l'histoire des conjonctions de subordination employées dans une cinquantaine de langues 
européennes, Kortmann (1997, cité par Bertin, 2003) «montre une tendance générale au passage d'un signe morphologiquement simple à un signe formellement complexe mais monovalent, la polyfonctionnalité sémantique et syntaxique étant inversement proportionnelle à la complexité morphologique » (Bertin, 2003: 271). Les formes polyvalentes sont plus sujettes au changement, changement qui va soit dans le sens d'une spécialisation, soit dans le sens de la disparition pure et simple de la forme en question.

Ajoutons enfin que au contraire qui est plus étoffé phonétiquement, plus explicite sémantiquement (la preuve en est son emploi absolu), puisqu'il est facilement rattachable à sa base lexicale (l'adjectif contraire), va être senti comme plus adéquat et plus approprié pour exprimer ce rapport d'incompatibilité entre les deux termes de l'opposition.

\section{Conclusion}

A la fin de son chapitre VI, dans lequel il oppose mais et ains, G. Antoine conclut : « Voilà au total, une liste de composés avec mais assez copieuse. Admettra-t-on, pour conclure, qu'elle présente un effort latent de notre langue pour réparer la perte de ains ?- Rien n'interdit de le penser; mais qui affirmera que le vide ait été comblé ? » (Antoine, 1958-1962 : 1157). Cette citation est la synthèse par laquelle il clôt une longue énumération des adverbes oppositifs employés en FM après mais afin de renforcer et rendre plus concrète l'opposition «trop abstraite », selon les termes d'Antoine, que cette conjonction permet d'exprimer. Pour notre part, nous avons rattaché, effectivement et comme le dit Antoine, cette disparition à une cause interne au système. La grammaticalisation de la locution adverbiale au contraire est à voir comme un changement lié à la disparition de ains. Ces deux changements sont apparemment indépendants, mais, comme nous espérons l'avoir démontré, peuvent être réunis sous une même cause interne au système grammatical, d'autant plus qu'ils se produisent pratiquement au même temps. Cette simultanéité des changements affectant des mots appartenant à une même catégorie sémantique ne peut être que significative. Le système de la langue tend naturellement à la simplification en mettant en oeuvre le principe d'économie et celui de l'iconicité. Le principe d'économie permet d'expliquer l'évincement de tout terme qui devient superflu vu l'apparition d'un autre qui peut le remplacer, et celui d'iconicité permet d'expliquer la préférence accrue des termes spécialisés. Par ailleurs, ces changements vont permettre une plus grande hiérarchisation des parties du discours, en offrant l'opposition conjonction/adverbe, lorsque la locution se combine avec mais, par contre, avec ains l'opposition était conjonction/conjonction et ne permettait donc aucune hiérarchisation. Cette hiérarchisation vers laquelle tend le système grammatical, permet aussi son évolution vers une plus grande simplification.

\section{Références bibliographiques}

Anscombre, J.-C. et Ducrot, O. (1977). Deux mais en français ?. Lingua n43, North Holland Publishing Company, p.23-40.

Antoine, G. (1958-1962). La coordination en français. Paris : d'Artrey, 1996.

Bertin, A. (2003). Les connecteurs de cause dans l'histoire du français - contradiction du changement linguistique. Verbum, fasc. 3, p.263-276

Combettes, B. (1995). Approche diachronique des adverbiaux contextuels. LINX, 3, p. 33-50.

Combettes, B. (2003). Au contraire, en revanche, par contre: aspects diachroniques. In Péroz, P. (éd) Contre : identité, sémantique et variation catégorielle.

Danjou-Flaux, N. (1983). Au contraire connecteur adversatif. Cahiers de linguistique française, 5, p.275-303.

Danjou-Flaux, N. (1986). Adversité et cohésion du discours. Modèles linguistiques, T. 8, 1, p.95-114.

Ducrot, O. (1984). Le dire et le dit. Paris

Heine, B. (2002). On the role of context in grammaticalization. New Reflections on grammaticalization, p.83-101. 
Kleiber, G. (1978). Sur l'emploi adversatif de mais et ainz (ainçois) en ancien français. Travaux de linguistique et de littérature, tome XVI, 1, p.271-292.

Marchello-Nizia, Ch. (2006). Grammaticalisation et changement linguistique, Bruxelles : De Boeck.

Meillet, A. (1982). Linguistique historique et linguistique générale. Coll. Linguistique, Paris VIII.

Melander, J. (1916). Etude sur MAGIS et les expressions adversatives dans les langues romanes. Upsal.

Rodriguez-Somolinos, A. (2000). Mais, ains, ainçois, en moyen français : syntaxe et sémantique. Le moyen français : La Recherche, Bilan et Perspectives, CERES, p.449-467.

Rodriguez-Somolinos, A. (2002). Ainz et mais en ancien français. Romania 477-478, tome 120, Société des amis de la Romania, Paris, p.505-541.

Vaugelas (de) C.-F. (1647). Remarques sur la langue françoise, Paris, la Veuve J. Camusat et P. le Petit, Genève : Slatkine reprints, 1970. ${ }^{1}$ Je tiens à exprimer ma reconnaissance et ma gratitude envers Madame Jacqueline Bacha pour son soutien et sa
disponibilité, elle a contribué à améliorer ce travail et tant d'autres. Je remercie également ma chère collègue
Thouraya Ben Amor pour ses remarques et suggestions pertinentes.
${ }^{2}$ Nous adoptons ici la graphie ains, mais il faut noter que ce mot se rencontre en AF sous différentes formes : anz,
ans, enz, ens, ainz, einz, eins, ains, ainc, et parfois ainques quand il n'est pas confondu avec onques. De plus, en
parlant de ains, nous incluons ainçois qui est, selon tous les linguistes qui se sont intéressés à cette conjonction, une
variante morphologique de ains. Ce mot se rencontre aussi sous des formes très diverses : ainçois, einceis, ançoires, anchois, anceis, ainceis.

${ }^{3}$ Nous croyons que les changements liés à la disparition de ains sont plus nombreux que ceux que nous venons d'évoquer, puisque nous pourrons inclure la grammaticalisation de l'adverbe plutôt et la locution conjonctive plutôt que mais nous ne pouvons nous intéresser, dans le cadre de l'article, ni à ces cas de grammaticalisation ni à celui de la spécialisation de mais.

${ }^{4}$ Nous empruntons ce terme et celui de 'macro-système' à Marchello-Nizia (2006)

${ }^{5}$ Pour la constitution de notre corpus, nous avons utilisé les trois bases de données suivantes :

- Pour l'AF, à partir du IX ${ }^{\mathrm{e}}$ siècle jusqu'à la fin du XIII ${ }^{\mathrm{e}}$ siècle nous avons utilisé la Base du Français Médiéval (La BFM) Cette base est accessible sur Internet à l'adresse suivante : http//bfm-ens-lsh.fr. Je profite de cette occasion pour remercier Alexei Lavrentiev qui gère la base et qui n'a jamais manqué de répondre à mes questions et mes demandes de détails aussi futiles soient-elles.

- $\quad$ Pour le MF, c'est-à-dire les XIV et XV siècles, nous avons utilisé le Dictionnaire du Moyen Français (le DMF). Cette base est accessible sur Internet à l'adresse suivante : http:// atilf.atilf.fr/dmf.

- $\quad$ Enfin, nous avons utilisé Frantext à partir du XVI ${ }^{\mathrm{e}}$ siècle.

${ }^{6}$ Ce mot est tantôt rattaché à l'adverbe temporel latin ante qui signifie « avant », tantôt à antius qui est un comparatif temporel du latin vulgaire au sens de "plus tôt », pour plus de détails voir à ce sujet Antoine (1958-1962:1118)

${ }^{7}$ Rodriguez- Somolinos a souligné à deux reprises cette ressemblance $(2000: 463)$ et $(2002: 528)$, de même pour G. Antoine (1958-1962: 1140).

${ }^{8}$ Pour plus de détails, voir Rodriguez-Somolinos (2000), en particulier p. 526 à 529.

${ }^{9}$ C'est pour cette raison que nous avons éliminé l'hypothèse du remplacement de ains par mais, les deux mots étant toujours restés sémantiquement différents. L'hypothèse du remplacement de ains par mais est retenue par Melander (1916) et par Rodriguez-Somolinos même si les deux linguistes admettent les différences sémantiques entre les deux coordonnants : «La distinction syntaxique une fois perdue, ains est senti comme superflu, il fait double emploi avec mais2 et s'efface peu à peu devant celui-ci » (Rodriguez-Somolinos, $2000: 466$ )

${ }^{10}$ Il ne s'agit pas d'un contraire dans le sens strict du terme mais plutôt d'un terme qui se trouve en relation d'incompatibilité avec le premier.

${ }^{11}$ Le premier terme de l'opposition comporte une négation sémantique, selon Danjou-Flaux, puisque « avoir honte d'effrayer quelqu'un implique qu'on renonce à le faire ». (Danjou-Flaux, $1986: 107$ ) 
${ }^{12}$ Des contraintes syntaxiques portent sur le type d'interrogation qui précède ains en $\mathrm{AF}$, ainsi que sur au contraire moderne. L'interrogation doit être totale, mais pour au contraire ce n'est pas suffisant, il faut qu'elle comporte obligatoirement une négation syntaxique ou lexicale, faute de quoi l'emploi de au contraire ne serait pas possible :

A- Ta voiture a-t-elle été réparée ? B- *Au contraire !

Par contre, on peut avoir :

A- Tu n'es pas contente de la réparation de ta voiture ? B- Au contraire !

Ces exemples sont empruntés à Danjou-Flaux (1986:96) et pour d'autres exemples se reporter à ce travail et à celui de 1983.

13 Combettes, B., 2003, a déjà étudié la grammaticalisation de la locution adverbiale au contraire, nous nous proposons dans ce travail d'élargir cette étude et de commencer l'enquête à partir des toutes premières apparitions du syntagme prépositionnel.

14 Nous remercions l'un de nos relecteurs anonymes qui nous a suggéré de mettre en parallèle la dimension typologique des textes du corpus avec les conditions d'emploi de au contraire et son évolution syntaxique, sémantique et énonciative.

${ }^{15}$ La préposition à est pratiquement la seule qui est employée avec le substantif contraire dans notre corpus, en effet nous n'avons repéré que 3 occurrences avec la préposition par et une seule fois avec en sous forme de l'article contracté ou dans un ensemble de 53 occurrences du substantif contraire, relevées dans la totalité du corpus de la BFM.

${ }^{16}$ Pour Combettes (2003: 278), le choix de la préposition à au détriment de par est motivé par « la valeur non marquée de à, apte à s'adapter à des contextes plus variés que par, en particulier à des contextes contenant des verbes statifs (être au contraire) [ce qui] peut expliquer que l'expression par contraire ne se soit pas maintenue. »

${ }^{17}$ Nous avons cherché uniquement les formes ainz, ains, eins, einz. Nous avons délibérément écarté les formes enz, anz et ainc d'abord parce qu'elles sont très rares, ensuite parce qu'elles peuvent avoir d'autres sens.

${ }^{18} \mathrm{Au} \mathrm{XVIII}^{\mathrm{e}}$ siècle, l'emploi de ains est extrêmement rare et archaïsant. Toutefois, au XIX ${ }^{\mathrm{e}}$ siècle, et même au début $\mathrm{du} \mathrm{XX}^{\mathrm{e}}$ siècle, certains auteurs comme Balzac, Musset, Stendhal, Sainte-Beuve, Flaubert et Bremond vont l'utiliser dans leurs écrits. Nous avons dénombré 9 occurrences au $\mathrm{XIX}^{\mathrm{e}}$ siècle et 2 au $\mathrm{XX}^{\mathrm{e}}$ siècle.

${ }^{19}$ Mais il ne s'agit pas uniquement de son emploi correspondant à celui de ains.

${ }^{20}$ Pour plus de détails concernant le fonctionnement oppositif de la locution au contraire, voir Danjou-Flaux (1983 et 1986)

${ }^{21}$ A part son fonctionnement en tant que coordonnant ains a gardé jusqu'au $\mathrm{XVI}^{\mathrm{e}}$ siècle un fonctionnement polyvalent, il est préposition ou adverbe signifiant «avant», locution prépositive : ainz de/que/que de + infinitif et signifiant soit «avant de » soit «plutôt» et locution conjonctive au sens de «avant que » lorsqu'elle est suivie d'un subjonctif, ou bien au sens de «plutôt que » si elle est suivie d'un indicatif.

${ }^{22}$ Les linguistes parlent d'isomorphisme ou bien de principe d'iconicité : un sens = une forme. 